\title{
EDITORIAL
}

\section{Next-generation strategies in animal model research to translate preclinical discoveries into better treatments for circuit-centered psychiatric dimensions}

\author{
Rodrigo Machado-Vieira, Carlos A. Zarate Jr. \\ Experimental Therapeutics and Pathophysiology Branch, Division of Intramural Research Program, National Institute of Mental Health, \\ National Institutes of Health, Bethesda, MD, USA.
}

The study of animal models is of key relevance in biomedical research. Animal models of psychiatric disorders have been important tools to gain insight into the presumed mechanisms of psychoses and mood and anxiety disorders in the last several decades. These models have largely focused on the improvement of specific aspects of validity. ${ }^{1}$ Our current psychopharmacological treatments came from these preclinical models for diverse psychiatric disorders. They provided not only a screening for identifying and further developing these compounds but also helped to clarify neurobiological aspects underlying psychiatric disorders. However, despite these gains, animal models have also contributed to the relatively little progress in developing new and improved therapeutics for human psychiatric disorders existing medications are largely refinements of older versions and have not been demonstrated to be superior in terms of efficacy.

A considerable number of our patients do not benefit from current medications and remain functionally impaired with poor quality of life, severely disabled, and at risk for suicidal behavior. Indeed, despite a critical need for new therapeutic targets for psychiatric disorders, we have been experiencing a period of less investment in drug development for psychiatric disorders than in previous decades. Several new compounds that seemed to satisfactorily improve phenotype in standard animal models were subsequently shown to have little impact in humans (phase I-Il studies). ${ }^{2}$ It is clear that some existing animal models are unable to mimic all symptoms of a specific disease, undoubtedly due in part to the multifaceted aspects of mimicking the subjective nature of human emotions, the complexity of higher human cognition, and the full-blown phenotype of the disorder. We also need to consider the environment and context in which animal model studies are conducted - how representative they are in the environment where humans live and socialize. Furthermore, transgenic animals evaluating specific genetic variants and alleles with small effect have been widely described and associated with specific phenotypes or neurobiological systems, but limited construct validity is still an issue due to the polygenic basis of psychiatric disorders.

Thus, some focus has shifted to developing the next generation of animal models. Recent research has highlighted the importance of developing new models based on circuit-centered dimensions (e.g., modeling "positive and negative affect" and "socioaffective function" for depression) to better understand disease rather than using current models of psychiatric classification. ${ }^{3}$ Our current psychiatric diagnoses probably represent a heterogeneous collection of disorders, each of which probably consists of many different disorders and etiologies. The use of newer models is likely to lead to a greater understanding of psychiatric disease than the approach which until now has largely relied on the study of behavioral phenotypes. Findings from clinical neuroscience can then be incorporated into the next generation of animal models.

We can also take advantage of research into treatments that are radically different from existing ones to better understand their mechanisms of action rather than using existing models to refine similar versions of treatments with limited efficacy. For example, the NMDA antagonist ketamine has been demonstrated to be a rapidly acting antidepressant in bipolar disorder and depression. ${ }^{4}$ Onset of antidepressant and antisuicidal action occurs within a few hours. Current antidepressants have an onset of 6 weeks or longer, and were largely developed from existing animal models. The finding of rapid antidepressant effects of ketamine in humans has translated back to preclinical research, referred to as "reverse engineering," in which animal models were used to identify several molecular targets implicated in its mechanism of action. This has permitted a more focused approach to honing in on the presumptive targets of a treatment, in stark contrast to traditional drug discovery and development models that have developed drugs with efficacy comparable to those of several decades ago on the basis of obscure notions of disease pathophysiology. Recent developments of noninvasive technologies for the study of human brain structure and function reinforce the need for translational studies in a two-way bridge (backtranslation), from bench to bedside and vice-versa.

Other exciting areas of study include the role of early stressors and epigenetics in the pathophysiology of mental disorders and investigations of endophenotypes in psychiatric disorders. These areas have been shown to properly translate animal models into valid human models of psychiatric disorders. ${ }^{5}$ Future research will need to 
take into account this line of research in animal model studies.

Another important aspect that requires further investigation in preclinical models is resilience to stress. ${ }^{6}$ This complex term describes a wide range of biological and psychosocial factors associated with successful adaptation and responses (or lack thereof) to stressors, such as early traumatic experiences. These involve cognitive flexibility, social support, physical activity, and other factors that account for individual variability in psychological responses and resilience to stress. Thus far, few animal models in psychiatry have been able to back-translate these aspects and incorporate them into valid animal models.

In conclusion, we are ready for the next generation of animal model research, where we will begin to incorporate better proxies of human emotions, cognition, and behaviors. We need to shift our focus to developing models that are circuit-centered and that take epigenetics and resilience into account. Finally, having drugs that are clearly superior in some aspects of treatment in humans will help focus the search at a mechanistic level and lead to improved therapeutics. These strategies, in a rapid and integrated back-and-forth approach from bench to bedside, will likely be used in future translational research and lead to greater insights into the pathophysiology of our diseases and to better treatments for circuit-centered psychiatric dimensions.

\section{Disclosure}

Carlos A. Zarate Jr. is listed as a coinventor on a patent application for the use of ketamine and its metabolites in major depression. He has assigned his rights in the patent to the US government but will share a percentage of any royalties that may be received by the government. Rodrigo Machado-Vieira has no conflicts of interest to disclose.

\section{References}

1 Machado-Vieira R, Kapczinski F, Soares JC. Perspectives for the development of animal models of bipolar disorder. Prog Neuropsychopharmacol Biol Psychiatry. 2004;28:209-24.

2 Machado-Vieira R. Tracking the impact of translational research in psychiatry: state of the art and perspectives. J Transl Med. 2012;10:175.

3 Berton O, Hahn CG, Thase ME. Are we getting closer to valid translational models for major depression? Science. 2012;338:75-9.

4 Zarate C Jr, Machado-Vieira R, Henter I, Ibrahim L, Diazgranados N, Salvadore G. Glutamatergic modulators: the future of treating mood disorders? Harv Rev Psychiatry. 2010;18:293-303.

5 Duman RS. Neuronal damage and protection in the pathophysiology and treatment of psychiatric illness: stress and depression. Dialogues Clin Neurosci. 2009;11:239-55.

6 Southwick SM, Charney DS. The science of resilience: implications for the prevention and treatment of depression. Science. 2012;338:79-82. 